\title{
How to increase the population of a Phlebotomus perniciosus (Diptera: Psychodidae) colony: a new method
}

\author{
Pedro María Alarcón-Elbal ${ }^{1 /+}$, Begoña García Montoliu' ${ }^{1}$, Rocío Pinal', Sarah Delacour-Estrella ${ }^{1}$, \\ Ignacio Ruiz-Arrondo', Miguel Ángel Peribáñez'1, Ignacio De Blas'1, Ricardo Molina², \\ Juan Antonio Castillo', Lorenzo Diéguez-Fernández ${ }^{3}$, Javier Lucientes ${ }^{1}$
}

${ }^{1}$ Departamento de Patología Animal, Facultad de Veterinaria, Universidad de Zaragoza, Zaragoza, Spain ${ }^{2}$ Unidad de Entomología Médica,
Servicio de Parasitología, Centro Nacional de Microbiología, Instituto de Salud Carlos III, Madrid, Spain
${ }^{3}$ Departamento de Control de Vectores, Unidad Municipal de Higiene y Epidemiología de Camagüey, Camagüey, Cuba

The sandfly Phlebotomus perniciosus is the most widespread vector of Leishmania infantum in Spain. Laboratory colonisation represents the most feasible source of information on the biology of these insects, but in conducting any study, the density of individuals in the colony may drop to such an extent that it is sometimes difficult to recover the initial population levels. A new technique was tested for the recovery of sandfly eggs in three different colonies; the recovery rate was studied by comparing the standard method of mass rearing with this new method of colony management. The results demonstrate a mean increase of $18.4 \%$ in adult production, a growth in colony productivity that justifies the inclusion of this process in the routine maintenance of any colony of sandflies.

Key words: Phlebotomus perniciosus - Leishmania infantum - sandfly - eggs recovery - colony productivity - Spain

Leishmaniases are a complex of worldwide diseases with a range of clinical and epidemiological features, which are caused by members of the genus Leishmania parasites that can infect numerous mammals, including humans (Gramiccia \& Gradoni 2005). These diseases have an important impact on public health, given the zoonotic nature of the process (WHO 1990), and are spread through sandflies of the genus Phlebotomus in the Old World and Lutzomyia in the New World.

In Spain, as throughout the western Mediterranean basin, the etiologic agent of leishmaniases is Leishmania infantum (Kinetoplastida: Trypanosomatidae), with dogs being the reservoir host (Alvar et al. 2004). Its transmission is seasonal and is related to the density of the sandfly population vectors and the length of their activity.

The bloodsucking females of Phlebotomus (Larrossius) perniciosus (Newstead, 1911) (Diptera: Psychodidae) are the most widespread vector of $L$. infantum in Spain (Aransay et al. 2003) and, in recent years, have also shown their capacity to transmit Toscana virus in the country (Sanbonmatsu-Gámez et al. 2005).

Maintaining a colony of $P$. perniciosus in the laboratory not only allows the possibility of studying the vector of L. infantum and searching for techniques to combat the disease by controlling the sandfly, but it also enables us to conduct studies of vectorial capacity, development of vaccines and xenodiagnosis. In conducting any ex-

Financial support: CICYT (AGL2007-66308-C02-02)

+Corresponding author: pedro.alarcon@uv.es

Received 4 April 2011

Accepted 22 July 2011 periment of this type, a high production of colony individuals is necessary. Due to the difficulty of breeding in captivity and the nature of these insects, it is sometimes difficult to recover the initial population levels. Because maintaining a colony is an ongoing job, it is important at each stage of sandfly breeding to assess methods to increase the colony population while taking into consideration the cost and the time involved.

Major difficulties in breeding sandflies in captivity include the high mortality of females after oviposition (Killick-Kendrick 1978, Maroli et al. 1987, Schlein et al. 1990) and fungal contamination (Marchais et al. 1991). It is therefore necessary to remove the bodies of dead sandflies (the main cause of fungal contamination in the medium) along with an excess of food for the larvae. This step is essential for the continuity of the colony, but results in a significant loss of eggs because many of them are glued to the bodies of sandflies that are removed. The retrieval of these eggs would thus afford an increase in adult production.

A new method for the recovery of eggs was tested. The recovery rate (RR) was studied by comparing the standard method of mass rearing combined with this new technique for colony maintenance.

\section{MATERIALS AND METHODS}

Field collection - Colonies of P. perniciosus reared in the Laboratory of Parasitology at the Universidad de Zaragoza were established with eggs obtained from females collected from three different locations: Madrid (Villaviciosa de Odón), Zaragoza (El Burgo de Ebro) and Huesca (Radiquero) (Figure). Sandflies from each of these regions were separated into different cages.

Colony maintenance - The tasks of mass rearing and maintenance of phlebotomine sandflies were carried out using the methods developed by Killick-Kend- 
rick et al. (1977), Endris et al. (1982), Molina (1991) and Benito de Martín (1997). The colonies were kept in an insectary maintained at $28 \pm 1^{\circ} \mathrm{C}, 95-100 \%$ relative humidity and 17:7 (L:D) photoperiod.

The holding cage for adult flies consisted of a $20 \times 20$ $\times 20 \mathrm{~cm}$ bag, with sides made of white cotton cloth and nylon organdie. The bag was suspended inside a $30 \mathrm{~cm}$ steel frame by string attached to its corners. Approximately 500 adult insects (equal ratio of males to females) were released into each cage. For feeding, a domestic rabbit (Oryctolagus cuniculus, California Breed) was anaesthetised with ketamine/xylazine (35/5 mg/kg given intramuscularly) and its head was carefully introduced in the holding cage through the sleeve. The choice of this animal for feeding was based on the fact that rabbits are one of the preferred hosts of P. perniciosus in our territory (Lucientes 1986). Cotton wool soaked in 10\% sucrose was placed on the top of the cage to provide a continuous source of water and sugar.

The containers for both mass rearing during immature stages and holding gravid females before oviposition were cylindrical $500 \mathrm{~mL}$ polymethylpentene jars (Nalgene, Genunc, cat. \#250865; Lima, OH, USA). The base of these jars was cut away and the bottom was filled with a layer of plaster ( $2 \mathrm{~cm}$ thick), while the original lid was replaced by a cotton cloth adapted to the jar mouth. Approximately 200 females were confined in each container for oviposition along with a similar quantity of males to ensure maximum fertilisation. The sandflies were fed using cotton saturated with a sucrose solution and each oviposition container was identified with a label indicating the date of blood ingestion.

Each week, the surviving individuals in the adult cages were released and the dead bodies were removed; otherwise, there would have been massive fungal contamination that could have endangered the viability of the emerging larvae. Removal was carried out with the aid of an entomological needle. After removing the adults, the cotton cloth covering the jar mouth was replaced by

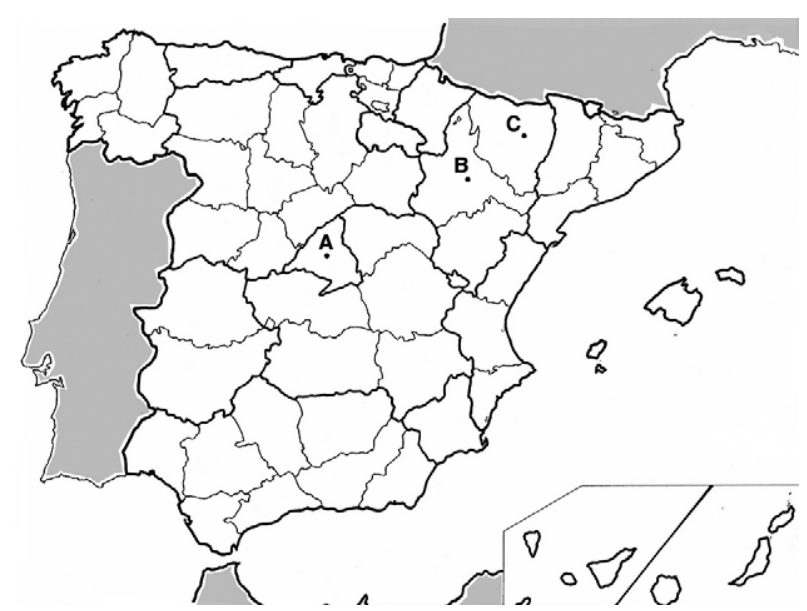

A: Villaciosa de Odón (Madrid); B: Burgo de Ebro (Zaragoza); C: Radiquero (Huesca). the jar lid and the oviposition jar became a rearing jar that only contained the eggs laid by the females.

After a period of incubation, which is approximately five days for P. perniciosus, the larvae 1 began to emerge. These larvae remained in the rearing jars throughout the four stages of development and during pupation until the emergence of the adults.

The larval diet contained equal amounts of rabbit faeces and rabbit food ground together (Modi \& Tesh 1983). After fermentation, this mixture was ground with a coffee grinder and then passed through an autoclave. Larvae were fed by sprinkling a thin layer of food over them. This part of the process was important, as too much food can cause death by suffocation, whereas contamination of the medium and insufficient food can lead to cannibalism amongst the larvae (Killick-Kendrick et al. 1977).

Recovery of eggs - To perform the test, the bodies of dead adults collected were kept in a conical polypropylene vial measuring $9 \mathrm{~cm}$ in height and $2.5 \mathrm{~cm}$ in diameter, with a screw cap (Sterilin, Feltham, England).

The vial was filled with distilled water at $90 \%$ capacity and was then closed with its corresponding cap. The content was mixed three times with a Vortex Reax 2000 (Heidolph Instruments GmbH \& Co KG, Schwabach, Germany) at 2,400 rpm for a period of $3 \mathrm{~s}$ each time. Reposing for 5 min after mixing, we obtained supernatant comprised of the remains of dead flies and sediment composed of the eggs. The supernatant was then transferred by decantation to another vial, ensuring that the sediment was not poured off. The operation was repeated again to ensure that the maximum number of eggs was retrieved.

The sediment was poured into a filter paper funnel. The eggs were then transferred back into their corresponding jar using a 3-mL Pasteur pipette $\left(\operatorname{Copan}^{\circledR}\right)$, which was used to apply water to the funnel to make the eggs fall into the jar. The eggs that were retrieved were stored in jars in the same way as those laid on plaster. The jars with the recovered eggs were marked with the date on which the females ingested blood and the letter $\mathrm{R}$; hence, each rearing jar had its corresponding recovered eggs jar.

The jars with the eggs (normal and recovered) were checked daily. To facilitate counting, the hatched adults in each jar were released into a new adult cage and were collected with a mouth aspirator.

Data analysis - The RR was determined by the formula $\mathrm{RR}=(\mathrm{RA} / \mathrm{NA}) \times 100$, where $\mathrm{RA}$ is the number of recovered adults and NA is the number of normal adults.

The difference in percentage of recovered eggs in the three colonies was analysed with the one-way analysis of variance model; data normality was checked with the Kolmogorov-Smirnov test. The accepted level of significance was established at $0.05 \%(\mathrm{p} \leq 0.05)$. The data were analysed with SPSS 15.0 for Windows (SPSS Inc, Chicago, IL, USA) software.

Ethics - The study was approved by the scientific committee of the Universidad de Zaragoza (PI12/10). 
TABLE

Efficiency (adult productivity) of the new technique for the recovery of sandfly eggs in three different sandfly colonies

\begin{tabular}{|c|c|c|c|c|c|c|c|c|c|}
\hline \multirow[b]{2}{*}{ Week } & \multicolumn{3}{|c|}{ Zaragoza } & \multicolumn{3}{|c|}{ Madrid } & \multicolumn{3}{|c|}{ Huesca } \\
\hline & $\begin{array}{l}\text { NA } \\
\text { (n) }\end{array}$ & $\begin{array}{l}\text { RA } \\
\text { (n) }\end{array}$ & $\begin{array}{l}\text { RR } \\
(\%)\end{array}$ & $\begin{array}{l}\text { NA } \\
\text { (n) }\end{array}$ & $\begin{array}{l}\text { RA } \\
\text { (n) }\end{array}$ & $\begin{array}{l}\text { RR } \\
(\%)\end{array}$ & $\begin{array}{l}\text { NA } \\
\text { (n) }\end{array}$ & $\begin{array}{l}\text { RA } \\
\text { (n) }\end{array}$ & $\begin{array}{l}\text { RR } \\
(\%)\end{array}$ \\
\hline 1 & 852 & 186 & 21.831 & 437 & 111 & 25.4 & 329 & 281 & 85.41 \\
\hline 2 & 1,147 & 236 & 20.575 & 1,183 & 226 & 19.104 & 1,254 & 176 & 14.035 \\
\hline 3 & 601 & 90 & 14.975 & 1,303 & 205 & 15.733 & 259 & 70 & 27.027 \\
\hline 4 & 531 & 34 & 6.403 & 771 & 116 & 15.045 & 720 & 66 & 9.167 \\
\hline 5 & 590 & 23 & 3.898 & 452 & 63 & 13.938 & 543 & 104 & 19.153 \\
\hline 6 & 502 & 126 & 25.1 & 1,454 & 218 & 14.993 & 318 & 79 & 24.843 \\
\hline 7 & 1,285 & 300 & 23.346 & 922 & 196 & 21.258 & 171 & 39 & 22.807 \\
\hline 8 & 871 & 177 & 20.321 & 1,260 & 439 & 34.841 & 501 & 100 & 19.96 \\
\hline 9 & 795 & 171 & 21.509 & 846 & 199 & 23.522 & 292 & 36 & 12.329 \\
\hline 10 & 163 & 16 & 9.816 & 1,111 & 197 & 17.732 & 894 & 196 & 21.924 \\
\hline 11 & 310 & 34 & 10.968 & 975 & 127 & 13.026 & 785 & 78 & 9.936 \\
\hline 12 & 1,099 & 132 & 12.011 & 1,077 & 128 & 11.885 & 930 & 91 & 9.785 \\
\hline Total & 8,746 & 1,525 & 17.436 & 11,791 & 2,225 & 18.87 & 6,996 & 1,316 & 18.811 \\
\hline
\end{tabular}

NA: normal adults; RA: recovered adults; RR: recovery rate in percentage.

\section{RESULTS}

For a 12-week period, one rearing jar per week was collected, with normal eggs deposited in each of the three colonies (Zaragoza, Madrid and Huesca). Using the techniques described here, the respective jar of recovered eggs was collected. There were a total of 72 jars from the three colonies, with 24 jars for each colony (12 jars with normal eggs and 12 with recovered eggs). Emerged adults from each jar were counted to observe the RR.

We calculated a RR of $17.44 \%$ for the Zaragoza colony, $18.87 \%$ for the Madrid colony and $18.40 \%$ for the Huesca colony (Table). There were no significant differences in the percentage or recovery eggs among the three colonies because the $\mathrm{p}$ value was greater than $0.05 \%(\mathrm{~F}=$ $0.89 ; \mathrm{df}=2,33 ; \mathrm{p}=0.42$ ).

A total of 32,599 adult sandflies hatched; 27,533 from the normal breeding jars and 5,066 from the recovered ones. The mean RR among the three colonies was $18.4 \%$.

\section{DISCUSSION}

Maintaining a sandfly colony in the laboratory provides the ability to study leishmaniasis in greater depth, including both the vector involved in its dispersal and the etiologic agent. However, this is a very complicated process because sandfly mortality during or after oviposition is a common phenomenon.

Performing an experiment in which a large number of sandflies is necessary can leave the colony with a very low population density. Maintaining the colony is an ongoing job; therefore, it is important to find ways to increase the population at each stage of breeding sandflies, taking into account the cost and the time involved. Studies by Maroli et al. (1987) showed that of all fe- males taking a first blood meal, only $70.7 \%$ laid eggs and $34.8 \%$ survived oviposition, and of these survivors, only $11.5 \%$ took a second blood meal. Under experimental conditions, sandflies usually die from exhaustion after the first oviposition. The females lay eggs on the plaster surface and on the side of the jar, but many eggs remain attached to the bodies of dead insects. It is thus necessary to remove the bodies of dead males and females after oviposition and release of the adult survivors to avoid contamination by fungi in rearing containers. In this process, a significant loss of eggs is noted, so the enhanced retrieval of these eggs would lead to an increase in the adult production.

The methods used in this study to recover eggs glued to the bodies of sandflies is based on the fact that the exoskeleton of these insects is composed of hydrocarbons; therefore, the insect bodies have a tendency to float in water, whereas their eggs form a denser layer of sediment. Thus, it is possible to practically recover all of the eggs glued to the dead insects. This process is entirely feasible to carry out once a week, which is the required frequency based on this experiment. Moreover, the economic cost and overall time involved is minimal.

The recovery of eggs by the method demonstrated in this paper represents a mean increase of $18.4 \%$ in the adult population of sandflies. This growth justifies the inclusion of this process in the routine maintenance of any colony of sandflies. This new method will be of great assistance to entomologists with sandfly colonies because populations are so slow to increase. It is also useful for other colonies in which testing causes a significant reduction in the population or for other species that are particularly difficult to rear. 


\section{REFERENCES}

Alvar J, Cañavate C, Molina R, Moreno J, Nieto J 2004. Canine leishmaniasis. Adv Parasitol 57: 1-88.

Aransay AM, Ready PD, Morillas-Márquez F 2003. Population differentiation of Phlebotomus perniciosus in Spain following postglacial dispersal. Heredity 90: 316-325.

Benito de Martín MI 1997. Phlebotomus perniciosus Newstead, 1911 en el Somontano de Barbastro: estudio entomológico y aspectos ecobiológicos en condiciones de laboratorio, $\mathrm{PhD}$ Thesis, Facultad de Veterinaria/Universidad de Zaragoza, Zaragoza, 355 pp.

Endris RG, Perkins PV, Young DG, Jonson RN 1982. Techniques for laboratory rearing of sandflies (Diptera, Psychodidae). Mosq News 42: 400-407.

Gramiccia M, Gradoni L 2005. The current status of zoonotic leishmaniases and approaches to disease control. Int J Parasitol 35: 1169-1180.

Killick-Kendrick R 1978. Recent advances and outstanding problems in the biology of phlebotomine sanflies. Acta Trop 35: 297-313.

Killick-Kendrick R, Leaney AJ, Ready PD 1977. The establishment, maintenance and productivity of a laboratory colony of Lutzomyia longipalpis (Diptera: Psychodidae). J Med Entomol 13: 429-440.

Lucientes J 1986. Contribución al conocimiento epidemiológico de la leishmaniosis visceral canina en Zaragoza: estudio biológico y ecológico de las poblaciones de los flebotomos vectores, $\mathrm{PhD}$ Thesis, Facultad de Veterinaria/Universidad de Zaragoza, Zaragoza, $424 \mathrm{pp}$.

Marchais R, Bounchet F, Bounchet P 1991. Laboratory rearing of Phlebotomus perniciosus (Diptera: Psychodidae) and fungal growth problems. Parassitologia 33 (Suppl.): 393-397.

Maroli M, Florentino S, Guandalini E 1987. Biology of a laboratory colony of Phlebotomus perniciosus (Diptera: Psychodidae). J Med Entomol 24: 547-551.

Modi GB, Tesh RB 1983. A simple technique for mass rearing Lutzomyia longipalpis and Phlebolomus papatasi (Diptera: Psychodidae) in the laboratory. J Med Entomol 20: 568-569.

Molina R 1991. Laboratory adaptation of an autochthonous colony of Phlebotomus perniciosus Newstead, 1911 (Diptera: Psychodidae). Res Rev Parasitol 51: 87-89.

Sanbonmatsu-Gámez S, Pérez-Ruiz M, Collao X, Sánchez-Seco MP, Morillas-Márquez F, de la Rosa-Fraile M, Navarro-Marí JM, Tenorio A 2005. Toscana virus in Spain. Emerg Infect Dis 11: 1701-1707.

Schlein Y, Borut S, Jacobson RL 1990. Oviposition diapause and other factors affecting the egg-laying of Phlebotomus papatasi in the laboratory. Med Vet Entomol 4: 69-78.

WHO - World Health Organization 1990. Control of the leishmaniases 1990. World Health Organ Tech Rep Ser 793: 1-177. 